\title{
MULTI-FOLLOWER TRI-LEVEL DECISION MAKING WITH UNCOOPERATIVE FOLLOWERS
}

\author{
JIALIN HAN ${ }^{1,2}$, JIE LU², GUANGQUAN ZHANG ${ }^{2}$, SHUYUAN MA ${ }^{1}$ \\ ${ }^{1}$ Industrial and Systems Engineering Laboratory, School of Mechanical Engineering, \\ Beijing Institute of Technology, China \\ ${ }^{2}$ Decision Systems and e-Service Intelligence Laboratory, Center for Quantum \\ Computation \& Intelligent Systems, Faculty of Engineering and Information Technology, \\ University of Technology, Sydney, Australia
}

\begin{abstract}
Multi-follower tri-level (MFTL) decision making addresses compromises among three interacting decision units within a hierarchical system of which multiple followers are involved in two lower-level units. The leader's decision is affected not only by reactions of the followers but also by various relationships among them. The uncooperative relationship is the most basic situation in MFTL decision cases where multiple followers at the same level make individual decisions without any information exchange or share among them. To support such a MFTL decision, this paper firstly proposes a general model for the decision problem and then develops an extreme-point search algorithm based on bi-level $K$ th-Best approach to solve the model. Finally, a numerical experiment illustrates the decision model and procedures of the extreme-point search algorithm.
\end{abstract}

\section{Introduction}

Tri-level decision making (also known as tri-level programming) can be considered as solving two optimization problems in sequence within a threelevel hierarchical system where the constraint region of the first is implicitly determined by the solution to the second that is a bi-level programming problem. This category of decision making often appears in many decentralized management problems with decision processes in a hierarchy in real world $[1,2]$ Decision entities allocated at the three hierarchical levels are respectively termed as the top-level leader, the middle-level follower and the bottom-level follower. Their decision processes are interactive until they achieve equilibrium in the vertical structure.

Tri-level decision making has been attracting increasing research on models $[3,4]$, solution concepts [3, 4], solution algorithms [3-5] and applications [6, 7]. However, the research has been limited to that one single decision entity is allocated at each level. Actually, multiple followers are often involved at the middle and bottom levels of a tri-level decision case called multi-follower trilevel (MFTL) decision making [8]. In this instance, the leader's decision is 
affected not only by reactions of multiple followers at both lower levels but also by various relationships among multiple followers at the same level. Trilevel decision making with such different relationships is needed to describe and solve by individual decision models and solution algorithms. However, investigates on hierarchical decision making with multiple followers have been centered on bi-level hierarchy [9-11] while nearly no research on MFTL decision making has been proposed except few brief programming models [8, 12]. Therefore, the research on MFTL decision making involving models, solution algorithms and applications should be further explored.

Within the framework of MFTL decision making, the uncooperative relationship is the most basic situation where multiple followers at the same level make their respective and individual decisions without any information exchange or share. This situation is very popular in a hierarchical organization involving competitive and uncooperative decision entities. Therefore, contributions of this paper aim to establish a general decision model and develop an available solution algorithm to describe and solve the uncooperative MFTL decision problem. More specifically, we firstly propose a linear MFTL decision model with the uncooperative relationship among both the middle-level followers and the bottom-level followers. To find a solution to the model, an extreme-point search algorithm based on bi-level Kth-Best approach [13] is developed. Finally, a numerical experiment is adopted to illustrate the MFTL decision model and procedures of the algorithm.

\section{An Uncooperative MFTL Decision Model}

For $x \in X \in R^{k}, y_{i} \in Y_{i} \subset R^{k_{i}}, Z_{i j} \in Z_{i j} \subset R^{k_{i j}}, F: X \times Y_{1} \times \cdots \times Y_{n} \times Z_{1} \times \cdots \times Z_{1 m_{1}} \times \cdots \times Z_{n m_{n}} \rightarrow R^{1}$, $f_{i}^{(2)}: X \times Y_{i} \times Z_{i 1} \times \cdots \times Z_{i m_{i}} \rightarrow R^{1}, f_{i j}^{(3)}: X \times Y_{i} \times Z_{i j} \rightarrow R^{1}, j=1,2, \cdots, m_{i}, i=1,2, \cdots, n$, an uncooperative MFTL decision model in a linear version in which one leader, $n$ middle-level followers and $m_{i}$ bottom-level followers attached to the $i$ th middlelevel follower are involved is defined as follows:

$$
\begin{aligned}
& \min _{x \in X} F\left(x, y_{1}, \cdots, y_{n}, z_{11}, \cdots, z_{1 m_{1}}, \cdots, z_{n 1}, \cdots, z_{n m_{n}}\right)=c x+\sum_{i=1}^{n} d_{i} y_{i}+\sum_{i=1}^{n} \sum_{j=1}^{m_{i}} e_{i j} z_{i j} \\
& \text { s.t. } A x+\sum_{i=1}^{n} B_{i} y_{i}+\sum_{i=1}^{n} \sum_{j=1}^{m_{i}} C_{i j} z_{i j} \leq b,
\end{aligned}
$$

where $\left(y_{i}, z_{i 1}, \cdots, z_{i m_{i}}\right)(i=1,2, \cdots, n)$ solves the $i$ th middle-level follower's and its bottom-level followers' problems (1c-1f):

$$
\min _{y_{i} \in Y_{i}} f_{i}^{(2)}\left(x, y_{i}, z_{i 1}, \cdots, z_{i m_{i}}\right)=c_{i} x+g_{i} y_{i}+\sum_{j=1}^{m_{i}} h_{i j} z_{i j}
$$




$$
\text { s.t. } A_{i} x+D_{i} y_{i}+\sum_{j=1}^{m_{i}} E_{i j} z_{i j} \leq b_{i}
$$

where $z_{i j}\left(j=1,2, \cdots, m_{i}\right)$ solves the $i$ th middle-level follower's $j$ th bottom-level follower's problem (1e-1f):

$$
\begin{aligned}
& \min _{z_{i j} \in Z_{i j}} f_{i j}^{(3)}\left(x, y_{i}, z_{i j}\right)=c_{i j} x+p_{i j} y_{i}+q_{i j} z_{i j} \\
& \text { s.t. } A_{i j} x+P_{i j} y_{i}+Q_{i j} z_{i j} \leq b_{i j},
\end{aligned}
$$

where $c, c_{i}, c_{i j} \in R^{k}, d_{i}, g_{i}, p_{i j} \in R^{k_{i}}, e_{i j}, h_{i j}, q_{i j} \in R^{k_{i j}}, A \in R^{s \times k}, A_{i} \in R^{s_{i} \times k}, A_{i j} \in R^{s_{i j} \times k}$, $B_{i} \in R^{s \times k_{i}}, D_{i} \in R^{s_{i} \times k_{i}}, P_{i j} \in R^{s_{i j} \times k_{i}}, C_{i j} \in R^{s \times k_{i j}}, E_{i j} \in R^{s_{i} \times k_{i j}}, Q_{i j} \in R^{s_{i j} \times k_{i j}}, b \in R^{s}, b_{i} \in R^{s_{i}}$, $b_{i j} \in R^{s_{i j}}$ for $j=1,2, \cdots, m_{i}, i=1,2, \cdots, n$.

\section{An Extreme-point Search Algorithm}

According to theoretical properties of multi-follower bi-level (MFBL) programming proposed by Calvete and Gale [9], we have the following theorem about MFTL decision making.

Theorem 1 The uncooperative MFTL decision problem (1) is equivalent to the MFTL decision problem (2) involving one leader, $n$ middle-level followers and one single bottom-level follower attached to each middle-level follower:

$$
\begin{aligned}
& \min _{x} F\left(x, y_{1}, \cdots, y_{n}, z_{11}, \cdots, z_{1 m_{1}}, \cdots, z_{n 1}, \cdots, z_{n m_{n}}\right)=c x+\sum_{i=1}^{n} d_{i} y_{i}+\sum_{i=1}^{n} \sum_{j=1}^{m_{i}} e_{i j} z_{i j} \\
& \text { s.t. } A x+\sum_{i=1}^{n} B_{i} y_{i}+\sum_{i=1}^{n} \sum_{j=1}^{m_{i}} C_{i j} z_{i j} \leq b,
\end{aligned}
$$

where $\left(y_{i}, z_{i 1}, \cdots, z_{i m_{i}}\right)(i=1,2, \cdots, n)$ solves the $i$ th middle-level follower's and its bottom-level followers' problems (2c-2f):

$$
\begin{aligned}
& \min _{y_{i}} f_{i}^{(2)}\left(x, y_{i}, z_{i 1}, \cdots, z_{i m_{i}}\right)=c_{i} x+g_{i} y_{i}+\sum_{j=1}^{m_{i}} h_{i j} z_{i j} \\
& \text { s.t. } A_{i} x+D_{i} y_{i}+\sum_{j=1}^{m_{i}} E_{i j} z_{i j} \leq b_{i},
\end{aligned}
$$

where $\left(z_{i 1}, \cdots, z_{i m_{i}}\right)$ solves the $i$ th middle-level follower's bottom-level follower's problem (2e-2f):

$$
\begin{aligned}
& \min _{z_{i 1}, \cdots, z_{i m_{i}}} f_{i}^{(3)}\left(x, y_{i}, z_{i 1}, \cdots, z_{i m_{i}}\right)=\sum_{j=1}^{m_{i}}\left(c_{i j} x+p_{i j} y_{i}+q_{i j} z_{i j}\right) \\
& \text { s.t. } A_{i j} x+P_{i j} y_{i}+Q_{i j} z_{i j} \leq b_{i j}, j=1,2, \cdots, m_{i} .
\end{aligned}
$$

\section{The extreme-point search algorithm}

Step 1: Set $k=1$, adopt the simplex method to obtain the optimal solution $\left(x^{1}, y_{1}^{1}, \cdots, y_{n}^{1}, z_{11}^{1}, \cdots, z_{1 m_{1}}^{1}, \cdots, z_{n 1}^{1}, \cdots, z_{n m_{n}}^{1}\right)$ to the linear programming problem 
$\left\{\min _{x} F\left(x, y_{1}, \cdots, y_{n}, z_{11}, \cdots, z_{1 m_{1}}, \cdots, z_{n 1}, \cdots, z_{n m_{n}}\right):\left(x, y_{1}, \cdots, y_{n}, z_{11}, \cdots, z_{1 m_{1}}, \cdots, z_{n 1}, \cdots, z_{n m_{n}}\right) \in S\right\}$. Let $W=\left\{\left(x^{1}, y_{1}^{1}, \cdots, y_{n}^{1}, z_{11}^{1}, \cdots, z_{1 m_{1}}^{1}, \cdots, z_{n 1}^{1}, \cdots, z_{n m_{n}}^{1}\right)\right\}$ and $T=\varnothing$. Set $i=1$ and go to Step 2 .

Step 2: Put $x=x^{k}$, and adopt the bi-level Kth-Best approach [13] to solve the $i$ th bi-level decision problem $(2 \mathrm{c}-2 \mathrm{f})$ and obtain the optimal solution $\left(\hat{y}_{i}, \hat{z}_{i 1}, \cdots, \hat{z}_{i m_{i}}\right)$. Then go to Step 3.

Step 3: If $\left(\hat{y}_{i}, \hat{z}_{i 1}, \cdots, \hat{z}_{i m_{i}}\right) \neq\left(y_{i}^{k}, z_{i 1}^{k}, \cdots, z_{i m_{i}}^{k}\right)$, go to Step 4. If $\left(\hat{y}_{i}, \hat{z}_{i 1}, \cdots, \hat{z}_{i m_{i}}\right)=$ $\left(y_{i}^{k}, z_{i 1}^{k}, \cdots, z_{i m_{i}}^{k}\right)$ and $i \neq n$, set $i=i+1$ and go to Step 2. If $\left(\hat{y}_{i}, \hat{z}_{i 1}, \cdots, \hat{z}_{i m_{i}}\right)=$ $\left(y_{i}^{k}, z_{i 1}^{k}, \cdots, z_{i m_{i}}^{k}\right)$ and $i=n$, stop and $\left(x^{k}, y_{1}^{k}, \cdots, y_{n}^{k}, z_{11}^{k}, \cdots, z_{1 m_{1}}^{k}, \cdots, z_{n 1}^{k}, \cdots, z_{n m_{n}}^{k}\right)$ is the optimal solution to the decision problems (2) and (1), and $K^{*}=k$.

Step 4: Let $W_{k}$ denote the set of adjacent extreme points of $\left(x^{k}, y_{1}^{k}, \cdots, y_{n}^{k}, z_{11}^{k}, \cdots, z_{1 m_{1}}^{k}, \cdots, z_{n 1}^{k}, \cdots, z_{n m_{n}}^{k}\right)$ such that $\left(x, y_{1}, \cdots, y_{n}, z_{11}, \cdots, z_{1 m_{1}}, \cdots, z_{n 1}, \cdots, z_{n m_{n}}\right) \in W_{k}$ implies $c x+\sum_{i=1}^{n} d_{i} y_{i}+\sum_{i=1}^{n} \sum_{j=1}^{m_{i}} e_{i j} z_{i j} \geq c x^{k}+\sum_{i=1}^{n} d_{i} y_{i}^{k}+\sum_{i=1}^{n} \sum_{j=1}^{m_{i}} e_{i j} z_{i j}^{k}$. Let $T=T \bigcup\left\{\left(x^{k}, y_{1}^{k}, \cdots, y_{n}^{k}\right.\right.$, $\left.\left.z_{11}^{k}, \cdots, z_{1 m_{1}}^{k}, \cdots, z_{n 1}^{k}, \cdots, z_{n m_{n}}^{k}\right)\right\}$ and $W=\left(W \cup W_{k}\right) \backslash T$. Go to Step 5.

Step 5: Set $k=k+1$ and choose $\left(x^{k}, y_{1}^{k}, \cdots, y_{n}^{k}, z_{11}^{k}, \cdots, z_{1 m_{1}}^{k}, \cdots, z_{n 1}^{k}, \cdots, z_{n m_{n}}^{k}\right)$ such that $c x^{k}+\sum_{i=1}^{n} d_{i} y_{i}^{k}+\sum_{i=1}^{n} \sum_{j=1}^{m_{i}} e_{i j} z_{i j}^{k}=\min \left\{c x+\sum_{i=1}^{n} d_{i} y_{i}+\sum_{i=1}^{n} \sum_{j=1}^{m_{i}} e_{i j} z_{i j}:\left(x, y_{1}, \cdots, y_{n}, z_{11}, \cdots, z_{1 m_{1}}, \cdots, z_{n 1}, \cdots\right.\right.$, $\left.\left.z_{n m_{n}}\right) \in W\right\}$. Set $i=1$ and go to Step 2 .

\section{A Numerical Experiment}

For $X=\{x: x \geq 0\}, Y_{i}=\left\{y_{i}: y_{i} \geq 0\right\}, Z_{i j}=\left\{z_{i j}: z_{i j} \geq 0\right\}, j=1,2, \cdots, m_{i}, i=1,2, m_{1}=m_{2}=2$, $\min _{x \in X} F\left(x, y_{1}, y_{2}, z_{11}, z_{12}, z_{21}, z_{22}\right)=-x+y_{1}-2 y_{2}+2 z_{11}+z_{12}-z_{21}-3 z_{22}$ s.t. $x+y_{1}+y_{2}+z_{11}+2 z_{12}+2 z_{21}+z_{22} \geq 9$, $x \leq 1$,

$\left\{\begin{array}{l}\min _{y_{1} \in Y_{1}} f_{1}^{(2)}\left(x, y_{1}, z_{11}, z_{12}\right)=x-y_{1}+z_{11}+z_{12} \\ \text { s.t. } 2 x+y_{1}+z_{11}+z_{12} \geq 8, \\ y_{1} \leq 1, \\ \left\{\begin{array}{c}\min _{z_{11} \in Z_{11}} f_{11}^{(3)}\left(x, y_{1}, z_{11}\right)=x+y_{1}+2 z_{11} \\ \text { s.t. } x+y_{1}+z_{11} \geq 4, \\ z_{11} \leq 3, \\ \min _{z_{12} \in Z_{12}} f_{12}^{(3)}\left(x, y_{1}, y_{0}, z_{12}\right)=2 x+y_{1}+z_{12} \\ \text { s.t. } x+y_{1}+z_{12} \geq 5, \\ x+z_{12} \leq 4,\end{array}\left\{\begin{array}{c}f_{2}^{(2)}\left(x, Y_{2}, z_{21}, z_{22}\right)=x+2 y_{2}+z_{21}+z_{22} \\ \text { s.t. } x+y_{2}+z_{21}+z_{22} \geq 4, \\ y_{2} \leq 1,\end{array}\right.\right. \\ \min _{z_{21} \in Z_{21}} f_{21}^{(3)}\left(x, y_{2}, z_{21}\right)=x+y_{2}+3 z_{21} \\ \text { s.t. } x+y_{2}+z_{21} \geq 2.5, \\ z_{21} \leq 1, \\ \min _{z_{22} \in Z_{22}} f_{22}^{(3)}\left(x, y_{2}, z_{21}\right)=x+2 y_{2}+2 z_{22} \\ \text { s.t. } x+y_{2}+z_{22} \geq 3, \\ z_{22} \leq 2 .\end{array}\right.$ 
We adopt the extreme-point search algorithm to solve the MFTL decision problem and the detailed computing process is showed as Table 1.

Table 1 The detailed computing process by the extreme-point search algorithm

\begin{tabular}{ccccc}
\hline Iteration $k$ & $s^{k}=\left(x^{k}, y_{1}^{k}, y_{2}^{k}, z_{11}^{k}, z_{12}^{k}, z_{21}^{k}, z_{22}^{k}\right)$ & $W_{k}$ & $T$ & $W$ \\
\hline 1 & $(1,1,1,2,3,1,2)$ & $\{(1,1,1,2,3,1,1)$, & $\left\{s^{1}\right\}$ & $W_{1}$ \\
& & $(0.5,1,1,2.5,3.5,1,2)$, & & \\
& & $(1,1,0.5,2,3,1,2)$, & & \\
3 & $(1,1,1,2,3,0.5,2)$ & $\{(1,1,1,1,2,3,0.5,1.5)\}$ & $\left\{s^{1}, s^{2}\right\}$ & $\left(W \cup W_{2}\right) \backslash T$ \\
4 & $(1,1,0.5,2,3,1,2)$ & $\{(1,1,0.5,2,3,1,1.5)\}$ & $\left\{s^{1}, s^{2}, s^{3}\right\}$ & $\left(W \cup W_{3}\right) \backslash T$ \\
5 & $(0.5,1,1,2.5,3.5,1,2)$ & $\{(0.5,1,1,2.5,3.5,1,1.5)\}$ & $\left\{s^{1}, s^{2}, s^{3}, s^{4}\right\}$ & $\left(W \cup W_{4}\right) \backslash T$ \\
6 & $(1,1,1,2,3,0.5,1.5)$ & $\varnothing$ & $\left\{s^{1}, s^{2}, s^{3}, s^{4}, s^{5}\right\}$ & $\left(W \cup W_{5}\right) \backslash T$ \\
& $(1,1,0.5,2,3,1,1.5)$ & & &
\end{tabular}

Table 1 presents the set $W_{k}$ of adjacent extreme points of each vertex $s_{k}$ after each iteration $k$. $T$ represents the set of extreme points that have been searched in the past iterations while $W$ is the set of extreme points that are needed to verify whether the optimal solution or not in the following iterations. We finally get the optimal solution through six iterations. In iteration 6 , $\left(x^{6}, y_{1}^{6}, y_{2}^{6}, z_{11}^{6}, z_{12}^{6}, z_{21}^{6}, z_{22}^{6}\right)=(1,1,0.5,2,3,1,1.5)$ is the optimal solution to the uncooperative MFTL decision problem and the objective function values of all decision entities are $F=0.5, f_{1}^{(2)}=5, f_{2}^{(2)}=4.5, f_{11}^{(3)}=6, f_{12}^{(3)}=6, f_{21}^{(3)}=4.5$, $f_{22}^{(3)}=5$. It is noticeable that $W_{5}=\varnothing$ in Table 1 does not mean adjacent extreme points of $\left(x^{5}, y_{1}^{5}, y_{2}^{5}, z_{11}^{5}, z_{12}^{5}, z_{21}^{5}, z_{22}^{5}\right)$ do not exist but implies its adjacent extreme points have been found in previous iterations and have been involved in $W$.

\section{Conclusions}

In a MFTL decision problem, various relationships among multiple followers at the same level would generate different decision processes. To support MFTL decision in an uncooperative situation, this paper firstly proposes a general decision model in a linear version. To find an optimal solution to the model, it then develops an extreme-point search algorithm based on bi-level Kth-Best approach [13]. Finally, a numerical experiment is adopted to illustrate the model and algorithm, which shows that the algorithm provides an available way in solving the proposed uncooperative MFTL decision problem. Our further research is to explore other relationships, such as cooperative and referenceuncooperative situations [8], among multiple followers and applications of MFTL decision making in real world. 


\section{References}

1. X. Xu, Z. Meng, and R. Shen, A tri-level programming model based on Conditional Value-at-Risk for three-stage supply chain management, Computers \& Industrial Engineering, 66, 470-475 (2013).

2. N. Alguacil, A. Delgadillo, and J. M. Arroyo, A trilevel programming approach for electric grid defense planning, Computers \& Operations Research, 41, 282-290 (2014).

3. J. F. Bard, An investigation of the linear three level programming problem, IEEE Transactions on Systems, Man, and Cybernetics, SMC-14, 711-717 (1984).

4. G. Zhang, J. Lu, J. Montero, and Y. Zeng, Model, solution concept, and Kth-best algorithm for linear trilevel programming, Information Sciences, 180, 481-492 (2010).

5. D. J. White, Penalty function approach to linear trilevel programming, Journal of Optimization Theory and Applications, 93, 183-197 (1997).

6. A. Street, A. Moreira, and J. M. Arroyo, Energy and reserve scheduling under a joint generation and transmission security criterion: An adjustable robust optimization approach, IEEE Transactions on Power Systems, 29, 314 (2013).

7. Y. Yao, T. Edmunds, D. Papageorgiou, and R. Alvarez, Trilevel optimization in power network defense, IEEE Transactions on Systems, Man, and Cybernetics, 37, 712-718 (2007).

8. J. Lu, G. Zhang, J. Montero, and L. Garmendia, Multifollower trilevel decision making models and system, IEEE Transactions on Industrial Informatics, 8, 974-985 (2012).

9. H. I. Calvete and C. Galé, Linear bilevel multi-follower programming with independent followers, Journal of Global Optimization, 39, 409-417 (2007).

10. B. Liu, Stackelberg-Nash equilibrium for multilevel programming with multiple followers using genetic algorithms, Computers \& Mathematics with Applications, 36, 79-89 (1998).

11. J. Lu, C. Shi, and G. Zhang, On bilevel multi-follower decision making: General framework and solutions, Information Sciences, 176, 1607-1627 (2006).

12. S. Hsu-Shih, L. Young-Jou, and E. S. Lee, Fuzzy approach for multi-level programming problems, Computers \& Operations Research, 23, 73-91 (1996).

13. J. F. Bard, Practical Bilevel Optimization: Algorithms and Applications. Dordrecht: Kluwer Academic Publishers, 195-204 (1998). 